\title{
S23. Long-term management of depressive disorders (supported by an educational grant from Lundbeck DK)
}

INDICATION FOR LONG TERM THERAPY

\section{OF DEPRESSIVE DISORDERS}

S. Kasper

University Hospital for Psychiatry, Department of General Psychiatry

A-1090 VIENNA, Währinger Gürtel 18-20, Austria

Clinicians mostly agree about the need to continue antidepressant medication until a current episode has resolved (continuation therapy). However, whether patients who have fully recovered should continue to receive medication in an attempt to prevent a future episode from taking place (maintenance therapy) is a more controversial one.

The data of recent longitudional studies indicate that mood disorders tend to be recurrent and lifetime in nature and that multiple episodes are the norm and not the exception. Furthermore, it has been found that the cycle length has a tendency to shorten with each new episode, and that the interval of being well between the first and second episodes was longer than the one between each subsequent set of episodes. Additionally, there is convincing evidence that increasing episodes excert an increasing risk factor for the occurance of another episode. A further important point, derived from epidemiological data is the finding that the older the patients are at the onset of the index episode, the more likely are early relapses if they remain untreated. Based on these findings it seems therefore that age of onset of first episode and number of prior episodes are prominent predictors for future episodes.

Although there is a lack of systematic studies to answer the question of when to begin maintenance therapy, there is clinical evidence that patients with more than three depressive episodes and those who are older than 50 years at first onset are at high risk of repeated depressive episodes. The number of phases and age of onset appear to be interactive, and it is probable that patients with more than three episodes (regardless of their age), and those who are aged over 50 years at the time of first onset are likely to benefit from maintenance treatment. Further variables (besides polarity, number of previous episodes, age of onset of first and index episode) which have been discussed to be predictive for a relapse in depression are: severity of index episode, work adjustment, social and leisure activity, gender, family history of depression and presence of other psychiatric or physical disorders (co-morbidity), like double depression (major depression + dysthymia), combined depression (major depression + recurrent brief depression).

\author{
MANAGEMENT OF CHRONIC, RECURRENT DEPRESSION \\ Stuart A Montgomery \\ St Mary's Hospital Medical School, London W2
}

Almost all depression is recurrent or chronic and strategies for treatment should aim to treat both acutely and chronically. European Guidelines recommend that in order to obtain a licence evidence of long term efficacy should be provided. All episodes of major depression require treatment beyond the point at which there is apparent response. If treatment is discontinued early in those who have a good symptomatic response, between $30 \%$ and $50 \%$ may expect their depressive symptoms to return. Placebo controlled studies with a variety of antidepressants have shown that this relapse rate is reduced with continued effective treatment and treatment should be continued for some 4 to 6 months after symptomatic response. Those who respond to placebo during the acute phase of treatment have also been shown to have a high early relapse rate and would appear to need effective continuation treatment. Recurrence prevention which aims to prevent new episodes of depression has been thoroughly studied. Imipramine is clearly effective but the most widely used antidepressant amitriptyline has for example insufficient convincing evidence of efficacy in this aspect of treatment. Two other tricyclic antidepressants which have been examined in placebo controlled studies in the prophylactic phase of treatment have convincing evidence of efficacy in doses which are in line with those shown to be effective in acute treatment. Recurrent brief depression, was found to be very common in the recent WHO field trials especially in general practice with a prevalence similar to that of major depression. As yet there is no evidence that conventional antidepressants are effective and the lack of efficacy of antidepressants in carefully conducted placebo controlled trials implies a possible different biological bias. The condition is seen commonly in the general population and general practice but surprisingly not in psychiatric practice which may reflect the lack of effective treatment. 
MAINTENANCE TREATMENT STRATEGIES IN RECURRENT UNIPOLAR DISORDERS

ㄴ Judd

Department of Psychiatry, University of CA at San Diego, 9500 Gilman Drive, La Jolla, CA 92093-0603

It has been established that major depressive disorders (MDD) are recurrent, and, most frequently, lifelong diseases. Even though the peak age onset for first episode of MDD is 25 years, episodes can occur at any age throughout the life cycle. There is a marked tendency for relapse in MDD, with over $80 \%$ of patients experiencing at least one more episode, and the average being 2 and 3 episodes. Approximately $30 \%$ of patients manifest a chronic clinical course with significant inter-episode symptomatology and disability.

The gradual acceptance of the recurrent nature of MDD has stimulated increasing research on long-term maintenance treatment strategies. Prien et al. reported significantly less relapse in unipolar depressives maintained on imipramine and lithium, and imipramine alone, in contrast to placebo or lithium alone. Glenn et al. report less relapse in severe recurrent unipolar depression with amitriptyline, as opposed to lithium or placebo. Montgomery et al. found that $74 \%$ successful prophylaxis in fluoxetine-treated unipolars in contrast to a $43 \%$ success on placebo. Kupfer et al, and Frank et al. have conducted the most systematic studies of maintenance strategies in recurrent unipolar depression published to date. They found a highly significant prophylactic effect on episode recurrence when patients are maintained at the same dose schedule for maintenance that was required to treat the original acute episode $(e . g$. $200 \mathrm{mg}$ of imipramine qd). In addition, they report delayed relapse and a modest prophylactic effect when monthly interpersonal psychotherapy (IPT) is added to the maintenance regimen. 